\title{
The Radius of Starlikeness of the Certain Classes of p-Valent Functions Defined by Multiplier Transformations
}

\author{
Mugur Acu, ${ }^{1}$ Yaşar Polatog̃lu, ${ }^{2}$ and Emel Yavuz ${ }^{2}$ \\ ${ }^{1}$ Department of Mathematics, "Lucian Blaga" University of Sibiu, 5-7 Ion Ratiu Street, \\ Sibiu 550012, Romania \\ ${ }^{2}$ Department of Mathematics and Computer Science, TC İstanbul Kültür University, \\ İstanbul 34156, Turkey
}

Correspondence should be addressed to Emel Yavuz, e.yavuz@iku.edu.tr

Received 12 November 2007; Accepted 02 January 2008

Recommended by Narendra Kumar K. Govil

The aim of this paper is to give the radius of starlikeness of the certain classes of $p$-valent functions defined by multiplier transformations. The results are obtained by using techniques of Robertson $(1953,1963)$ which was used by Bernardi (1970), Libera (1971), Livingstone (1966), and Goel (1972).

Copyright (c) 2008 Mugur Acu et al. This is an open access article distributed under the Creative Commons Attribution License, which permits unrestricted use, distribution, and reproduction in any medium, provided the original work is properly cited.

\section{Introduction}

Let $\mathscr{H}$ be the class of analytic functions in the open unit disc $\mathbb{D}=\{z \in \mathbb{C}|| z \mid<1\}$ and $\mathscr{H}[a, n]$ be the subclasses of $\mathscr{H}$ consisting of the functions of the form $f(z)=a+a_{n} z^{n}+a_{n+1} z^{n+1} \ldots$. Let $\mathcal{A}(p, n)$ denote the class of functions $f(z)$ normalized by

$$
f(z)=z^{p}+\sum_{k=n+p}^{\infty} a_{k} z^{k} \quad(p, n \in \mathbb{N}:=\{1,2,3, \ldots\})
$$

which are analytic in the open unit disc $\mathbb{D}$. In particular, we set

$$
\mathcal{A}(p, 1):=\mathcal{A}_{p}, \quad \mathcal{A}(1,1):=\mathcal{A}=\mathscr{A}_{1} .
$$

If $f(z)$ and $g(z)$ are analytic in $\mathbb{D}$, we say that $f(z)$ is subordinate to $g(z)$, written symbolically as

$$
f \prec g \quad \text { or } \quad f(z) \prec g(z) \quad(z \in \mathbb{D}) \text {. }
$$


If there exists a Schwarz function $w(z)$ which is analytic in $\mathbb{D}$ with $w(0)=0,|w(z)|<1$ such that $f(z)=g(w(z)), z \in \mathbb{D}$.

For two analytic functions $f(z)$ and $F(z)$, we say that $F(z)$ is superordinate to $f(z)$ if $f(z)$ is subordinate to $F(z)$.

For integer $n \geq 1$, let $\Omega(n)$ denote the class of functions $w(z)$ which are regular in $\mathbb{D}$ and satisfy the conditions $w(0)=0,|w(z)|<1$, and $w(z)=z^{n} \phi(z)$ for all $z \in \mathbb{D}$, where $\phi(z)$ is regular and analytic in $\mathbb{D}$ and satisfies $|\phi(z)|<1$ for every $z \in \mathbb{D}$. Also, let $D\{(p, n)$ denote the class of functions $p(z)=p+\sum_{k=n}^{\infty} p_{k} z^{k}$ which are regular in $\mathbb{D}$ and satisfy the conditions $p(0)=p, \operatorname{Re} p(z)>0$ for all $z \in \mathbb{D}$. We note that if $p(z) \in p(p, n)$, then

$$
p(z)=p \frac{1-w(z)}{1+w(z)}=\frac{1-z^{n} \phi(z)}{1+z^{n} \phi(z)}
$$

for some functions $w(z) \in \Omega(n)$ and every $z \in \mathbb{D}$.

Definition 1.1. Let $f(z) \in \mathcal{A}(p, n)$ for $m \in \mathbb{N}_{0}=\mathbb{N} \cup\{0\}, \lambda \geq 0, l>0$, one defines the multiplier transformations $\supset_{p}(m, \lambda, l)$ on $\mathcal{A}(p, n)$ by the following infinite series:

$$
\supset_{p}(m, \lambda, l) f(z):=z^{p}+\sum_{k=p+n}^{\infty}\left(\frac{p+\lambda(k-p)+l}{p+l}\right)^{m} a_{k} z^{k} .
$$

It follows that

$$
\begin{gathered}
\supset_{p}(0, \lambda, l) f(z)=f(z), \\
(p+l) \supset_{p}(2, \lambda, l) f(z)=(p(1-\lambda)+l) \supset_{p}(1, \lambda, l) f(z)+\lambda z\left(\supset_{p}(1, \lambda, l) f(z)\right)^{\prime}, \\
\supset_{p}\left(m_{1}, \lambda, l\right)\left(\supset_{p}\left(m_{2}, \lambda, l\right) f(z)\right)=\supset_{p}\left(m_{2}, \lambda, l\right)\left(\supset_{p}\left(m_{1}, \lambda, l\right) f(z)\right)
\end{gathered}
$$

for all integers $m_{1}, m_{2}$.

Remark 1.2. This multiplier transformation was introduced by Cătaş [1]. For $p=1, l=0, \lambda \geq 0$, the operator $\Phi_{\lambda}^{m}:=\supset_{1}(m, \lambda, 0)$ was introduced by Al-Oboudi [2] which reduces to the Sălăgean differantial operator [3]. For $\lambda=1$, the operator $\supset_{l}^{m}:=\supset_{1}(m, 1, l)$ was studied recently by Cho and Srivastava [4] and Cho and Kim [5]. The operator $\supset_{m}:=\supset_{1}(m, 1,1)$ was studied by Uralegaddi and Somanatha [6] and the operator $\supset_{p}(m, l):=\supset_{p}(m, 1, l)$ was investigated recently by Sivaprasad Kumar et al. [7].

Definition 1.3 (see [1]). Let $\varphi(z)$ be analytic in $\mathbb{D}$ and $\varphi(0)=1$. A function $f(z) \in \mathcal{A}(p, n)$ is said to be in the class $A_{p}(m, \lambda, l, n ; \varphi)$ if it satisfies the following subordination:

$$
\frac{\supset_{p}(m+1, \lambda, l) f(z)}{\supset_{p}(m, \lambda, l) f(z)} \prec \varphi(z) \quad(z \in \mathbb{D}) .
$$

Definition 1.4. The radius of starlikeness of the class $\mathcal{A}_{p}(m, \lambda, l, n, \varphi)$ is defined by the following. For each $f(z) \in \mathcal{A}_{p}(m, \lambda, l, n ; \varphi)$, let $r(f)$ be the supremum of all numbers $r$ such that $f\left(\mathbb{D}_{r}\right)$ is starlike with respect to the origin. Then the radius of starlikeness for $\mathcal{A}_{p}(m, \lambda, l, n ; \varphi)$ is

$$
r_{\mathrm{st}}\left(\mathcal{A}_{p}(m, \lambda, l, n ; \varphi)\right)=\inf _{f \in \mathcal{A}_{p}(m, \lambda, l, n, \varphi)} r(f) .
$$


Mugur Acu et al.

Theorem 1.5. Let $f(z) \in \mathcal{A}(p, n)$ and $\lambda>0$, then $f(z)$ belongs to the class $\mathcal{A}_{p}(m, \lambda, l, n ; x)$ if and only if $F(z)$, defined by

$$
F(z)=\frac{p+l}{\lambda z^{(p(1-\lambda)+l) / \lambda}} \int_{0}^{z} \zeta^{(p(1-\lambda)+l) / \lambda-1} f(\zeta) d \zeta=z^{p}+\sum_{k=p+n}^{\infty}\left(\frac{p+l}{p+l+(k-p) \lambda}\right) a_{k} z^{k}
$$

belongs to the class $\mathcal{A}_{p}(m+1, \lambda, l, n ; \chi)$.

This theorem was proved by Cătaş [1].

\section{Main result}

Theorem 2.1. The radius of starlikeness of the class $\mathcal{A}_{p}(m, \lambda, l, n, \phi)$ is

$$
r_{\mathrm{st}}=\left(\frac{p+l}{\lambda(p+n)+\sqrt{\lambda^{2}(p+n)^{2}+(p+l)(p+l-2 \lambda p)}}\right)^{1 / n} .
$$

This radius is sharp because the extremal function is

$$
f_{*}(z)=\frac{\lambda}{p+l} \frac{z^{p}\left(c+p+(c-p) z^{n}\right)}{\left(1+z^{n}\right)^{2 p / n+1}}, \quad c=\frac{p(1-\lambda)+l}{\lambda} .
$$

Proof. If we take $c=(p(1-\lambda)+l) / \lambda$, then the function $F(z)$ in Theorem 1.5 can be written in the form

$$
F(z)=\frac{p+l}{\lambda z^{c}} \int_{0}^{z} \zeta^{c-1} f(\zeta) d \zeta
$$

If we take the logarithmic derivative from (2.3) and after simple calculations, we get

$$
z \frac{F^{\prime}(z)}{F(z)}=\frac{z^{c} f(z)-c \int_{0}^{z} \zeta^{c-1} f(\zeta) d \zeta}{\int_{0}^{z} \zeta^{c-1} f(\zeta) d \zeta}
$$

Since $F(z)$ is starlike, hence there exists a function $w(z) \in \Omega(n)$ such that

$$
z \frac{F^{\prime}(z)}{F(z)}=\frac{z^{c} f(z)-c \int_{0}^{z} \zeta^{c-1} f(\zeta) d \zeta}{\int_{0}^{z} \zeta^{c-1} f(\zeta) d \zeta}=p \frac{1-w(z)}{1+w(z)}
$$

Solving for $f(z)$,

$$
f(z)=\frac{(c+p)+(c-p) w(z)}{(1+w(z)) z^{c}} \int_{0}^{z} \zeta^{c-1} f(\zeta) d \zeta
$$


Taking the logarithmic derivative from (2.6), we get

$$
z \frac{f^{\prime}(z)}{f(z)}=p \frac{1-w(z)}{1+w(z)}+(b-1) \frac{z w^{\prime}(z)}{(1+w(z))(1+b w(z))},
$$

where $b=(c-p) /(c+p)$. To show that $f(z)$ is starlike in $|z|<r_{0}$, we must show that

$$
\operatorname{Re}\left(z \frac{f^{\prime}(z)}{f(z)}\right)>0
$$

for $|z|<r_{0}$. This condition is equivalent to

$$
(1-b) \operatorname{Re}\left(\frac{z w^{\prime}(z)}{(1+w(z))(1+b w(z))}\right) \leq \operatorname{Re}\left(p \frac{1-w(z)}{1+w(z)}\right) .
$$

On the other hand, we have the following relations:

$$
\begin{gathered}
\operatorname{Re}\left(p \frac{1-w(z)}{1+w(z)}\right)=p \frac{1-|w(z)|^{2}}{|1+w(z)|^{2}}, \\
(1-b) \operatorname{Re}\left(\frac{z w^{\prime}(z)}{(1+w(z))(1+b w(z))}\right) \leq \frac{(1-b)\left|z w^{\prime}(z)\right|}{|1+w(z)||1+b w(z)|^{\prime}}, \\
\left|z w^{\prime}(z)\right| \leq \frac{n|z|^{n}}{1-|z|^{2 n}}\left(1-|w(z)|^{2}\right)
\end{gathered}
$$

(Golusin inequality, [8]). Therefore, the inequality (2.9) will be satisfied if

$$
\frac{n(1-b)|z|^{n}}{|1+w(z)||1+b w(z)|} \frac{1-|w(z)|^{2}}{1-|z|^{2 n}} \leq p \frac{1-|w(z)|^{2}}{|1+w(z)|^{2}} .
$$

Simplifying and writing $|z|=r$, we obtain

$$
\frac{n(1-b) r^{n}}{1-r^{2 n}} \leq p\left|\frac{1+b w(z)}{1+w(z)}\right|
$$

Since $|w(z)| \leq|z|^{n}=r^{n}, p|(1+b w(z)) /(1+w(z))| \geq p\left(\left(1+b r^{n}\right) /\left(1+r^{n}\right)\right)$ so that $(2.12)$ will be satisfied if

$$
\frac{n(1-b) r^{n}}{1-r^{2 n}}<p \frac{1+b r^{n}}{1+r^{n}}
$$

The inequality (2.13) can be written in the following form:

$$
p-(1-b)(p+n) r^{n}-b p r^{2 n}>0,
$$

which gives the required root $r_{0}$ of the theorem.

To see that the result is sharp, consider the function $F(z)=z^{p} /\left(1+z^{n}\right)^{2 p / n}$. For this function, we have

$$
\begin{aligned}
f_{*}(z) & =\frac{\lambda}{p+l} \frac{z^{p}\left((c+p)+(c-p) z^{n}\right)}{\left(1+z^{n}\right)^{2 p / n+1}}, \\
z \frac{f_{*}^{\prime}(z)}{f_{*}(z)} & =\frac{p-(1-b)(p+n) z^{n}-p b z^{2 n}}{\left(1+z^{n}\right)^{2 p / n+1}} .
\end{aligned}
$$

So that $z\left(f_{*}^{\prime}(z) / f_{*}(z)\right)=0$ for $|z|=r_{0}$. Thus, $f(z)$ is not starlike in any circle $|z|<r$ if $r>r_{0}$. 
Remark 2.2. If we give special values to $m, \lambda, l, n$, we obtain the radius of starlikeness for the corresponding integral operators.

\section{Acknowledgment}

This paper was supported by GAR 20/2007.

\section{References}

[1] A. Cătaş, On certain classes of $p$-valent functions defined by multiplier transformations, in Proceedings of the International Symposium on Geometric Function Theory and Applications, İstanbul, Turkey, August 2007.

[2] F. M. Al-Oboudi, On univalent functions defined by a generalized Sălăgean operator, International Journal of Mathematics and Mathematical Sciences, vol. 2004, no. 27, pp. 14291436, 2004.

[3] G. S. Sălăgean, Subclasses of univalent functions, in Complex Analysis_Fifth Romanian-Finnish Seminar, Part 1 (Bucharest, 1981), vol. 1013 of Lecture Notes in Math, pp. 362372, Springer, Berlin, Germany, 1983.

[4] N. E. Cho and H. M. Srivastava, Argument estimates of certain analytic functions defined by a class of multiplier transformations, Mathematical and Computer Modelling, vol. 37, no. 1-2, pp. 3949, 2003.

[5] N. E. Cho and T. H. Kim, Multiplier transformations and strongly close-to-convex functions, Bulletin of the Korean Mathematical Society, vol. 40, no. 3, pp. 399410, 2003.

[6] B. A. Uralegaddi and C. Somanatha, Certain classes of univalent functions, in Current Topics in Analytic Function Theory, pp. 371374, World Scientific, Singapore, 1992.

[7] S. Sivaprasad Kumar, H. C. Taneja, and V. Ravichandran, Classes of multivalent functions defined by Dziok-Srivastava linear operator and multiplier transformation, Kyungpook Mathematical Journal, vol. 46, no. 1, pp. 97109, 2006.

[8] G. M. Golusin, Geometrische Funktionentheorie, vol. 31 of Hochschulbücher für Mathematik, VEB Deutscher Verlag der Wissenschaften, Berlin, Germany, 1957. 